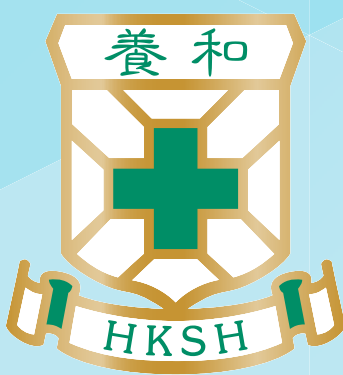

\title{
Elucidating uncertainties in radiobiological parameters in proton beam irradiation by a global fit
}

\author{
Hervé Hiu Fai Choi, Chen-Yu Huang, Wing Kei Rebecca Wong, Yick Wing Ho, \\ Kin Yin Cheung, Siu Ki Yu \\ Medical Physics \& Research Department, Hong Kong Sanatorium \& Hospital, Hong Kong \\ Corresponding author: Hervé Choi (herve.hf.choi@hksh.com)
}

\section{Purpose}

Relative biological effectiveness (RBE) of a proton beam has been taken to be 1.1 relative to Co-60 for current treatment planning; however, numerous works have shown that the RBE for a proton beam is far from uniform. Rather, as the proton beam transverses a medium, its linear energy transfer (LET) changes before it stops and the RBE increases correspondingly. Other radiobiological parameters such as $\alpha / \beta$ have also been found to vary with respect to LET. A uniform RBE factor may thus be inadequate in predicting the tumour response and the toxicity to the peripheral normal tissues. Better understanding of RBE as a function of LET is therefore crucial in ensuring the safety and efficacy of proton therapy treatment.

Recently, Abolfath et al. [1] has proposed a global fit to cellular survival curves across different LETs to reduce uncertainties in the fit and elucidate unphysical fluctuations of $\alpha / \beta$ in the function of LET. We applied their global fit on published datasets of V79 Chinese hamster lung fibroblasts and AG01522 normal human skin fibroblasts under proton beam irradiation and re-analyzed them with a single global fit across all LETs.

\section{Method}

Cell survival data as a function of dose with different LETs were extracted from studies [2], [3], [4], and [5]. A summary of the data is in Table 1.

Table 1: Summary of radiobiological experiments used in this work, along with the corresponding LET range

\begin{tabular}{|l|l|l|}
\hline Study & Cell line & LET range $(\mathrm{keV} / \mu \mathrm{m})$ \\
\hline Belli et al. [2] & V79 & $7.0-37.8$ \\
\hline Folkard et al. [3] & V79 & $10-28$ \\
\hline Wouters et al. [4] & V79 & $2.2-5.7$ \\
\hline Carante et al. [5] & AG01522 & $1.1-25.9$
\end{tabular}

The data points were then fitted using linear least squares in Matlab (MathWorks, Natick, MA USA). The fitting function is:

$$
-\log _{10}(S F)=\sum_{i=1}^{5} \sum_{j=1}^{i} p_{i j} D^{j} L E T^{i-j}
$$

where SF is the cell survival fraction, $p_{i j}$ are the fitting parameters, and $D$ is the dose. The difference between Abolfath's natural log and our fit is a factor of $\ln (10)$ and will be absorbed in the values of $p_{i j}$. Radiobiological parameters $\alpha$ and $\beta$ can be extracted from the fit as a function of LET:

$$
\alpha=\sum_{i=1}^{5} p_{i 1} L E T^{i} \quad \beta=\sum_{i=1}^{3} p_{i 2} L E T^{i}
$$

To avoid unphysical fitting results, we restricted $p_{i 2}$ to be non-negative.

\section{Results}

The fit to the data points appear reasonable, with the adjusted $R^{2}$ of the fits for V79 and AG01522 being 0.9754 and 0.9772, respectively. The RBE and the $\alpha / \beta$ ratio calculated from the fit as a function of LET display a smooth trend.

For the V79 data, the $\alpha / \beta$ calculated with the global fit peaks at LET $30 \mathrm{keV} / \mu \mathrm{m}$. The $\alpha / \beta$ was not calculated in the LET $>30 \mathrm{keV} / \mu \mathrm{m}$ region in Belli since the quadratic term did not yield a better fit.
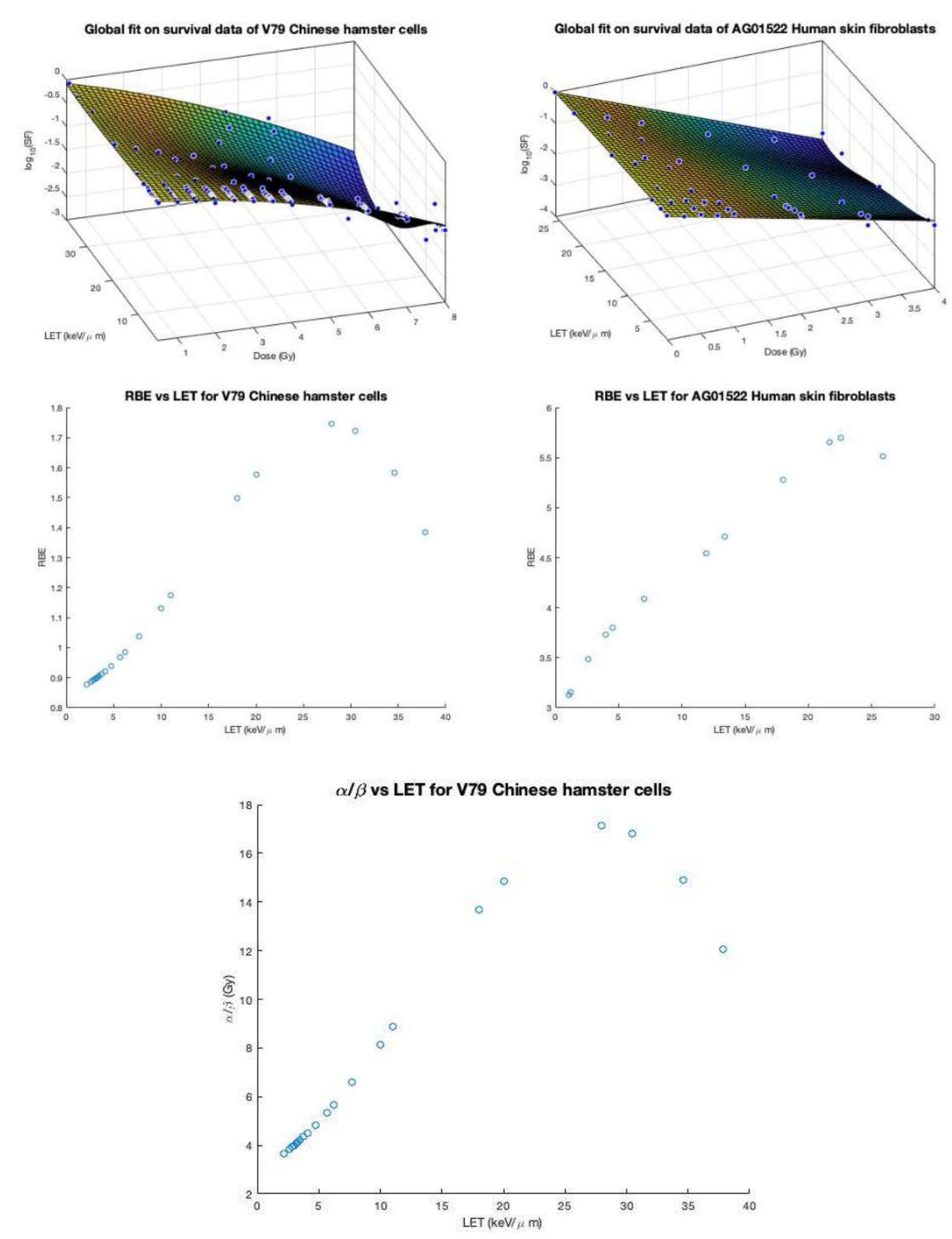

\section{Conclusions}

The global fit technique can be useful to elucidate uncertainties across different LETs. It can also facilitate determination of values for radiobiological parameters that may otherwise be undeterminable. This can lead to better understanding of biological response of tissues near the end of the proton range.

\section{References}

1. R Abolfath, CR Peeler, M Newpower, L Bronk, D Grosshans and R Mohan. "A model for relative biological effectiveness of therapeutic proton beams based on a global fit of cell survival data." Sci Rep 7:8340, 2017.

2. M Belli, F Cera, R Cherubini et al. "RBE-LET relationships for cell inactivation and mutation induced by low energy protons in V79 cells: further results at the LNL facility." Int J Radiat Biol 74(4): 501-509, 2017.

3. M Folkard, KM Prise, B Vojnovic, HC Newman, MJ Roper and BD Michael. "Inactivation of V79 cells by low-energy protons, deuterons and helium-3 ions." Int J Radiat Biol 69(6): 729-738, 1996.

4. BG Wouters, GKY Lam, U Oelfke et al. "Measurements of relative biological effectiveness of the $70 \mathrm{MeV}$ proton beam at TRIUMF using Chinese hamster V79 cells and the high-precision cell sorter assay." Radiat Res 146: 159-170, 1996.

5. MP Carante and F Ballarini. "Calculating variations in biological effectiveness for a $62 \mathrm{MeV}$ proton beam." Front Oncol 6: 76, 2016. 